This item was submitted to Loughborough's Research Repository by the author.

Items in Figshare are protected by copyright, with all rights reserved, unless otherwise indicated.

\title{
Assessing wireless inertia measurement units for monitoring athletics sprint performance
}

PLEASE CITE THE PUBLISHED VERSION

http://dx.doi.org/10.1109/ICSENS.2014.6985476

\section{PUBLISHER}

(C) 2014 IEEE

\section{VERSION}

AM (Accepted Manuscript)

\section{PUBLISHER STATEMENT}

This work is made available according to the conditions of the Creative Commons Attribution-NonCommercialNoDerivatives 4.0 International (CC BY-NC-ND 4.0) licence. Full details of this licence are available at: https://creativecommons.org/licenses/by-nc-nd/4.0/

\section{LICENCE}

CC BY-NC-ND 4.0

\section{REPOSITORY RECORD}

Philpott, Lydia K., Sam Weaver, David Gordon, Paul P. Conway, and Andrew A. West. 2019. "Assessing Wireless Inertia Measurement Units for Monitoring Athletics Sprint Performance". figshare. https://hdl.handle.net/2134/18607. 


\title{
Assessing wireless inertia measurement units for monitoring athletics sprint performance.
}

\author{
Lydia K. Philpott, Sam Weaver, D. Gordon, Paul P. Conway and Andrew A. West \\ Wolfson School of Mechanical and Manufacturing Engineering, Loughborough University, \\ Loughborough, UK \\ Email: 1.k.philpott@lboro.ac.uk
}

\begin{abstract}
A cheap and easy to use monitoring device that can be used within the athletics sprinting community is being developed. A device which enables coaches to monitor key features of an athlete's sprint start, allowing the effect of any technical adjustments made to be examined closely, could be used as a tool to assist in an athlete's development of a more optimsed performance. Whilst wireless inertial measurement units have been used successfully to monitor running, walking and to recognise other day to day activities such as stair walking, sitting and driving, the efficacy of these devices to be used in the monitoring of a sprint start in a running race has yet to be determined. The accuracy of both the timing and acceleration profiles recorded by a wireless wearable device, when compared to data collected using Vicon motion capture system, are described in this paper. Twenty five sprint start trials were recorded and eight features where identified and automatically determined via bespoke software algorithms. Timing and acceleration data from these points were then compared between the two methods of collection. The timing accuracy of the inertial measurement unit was accurate to $0.025 \pm 0.024 \mathrm{~s}$. The variance between the acceleration readings was larger ranging from 1.15 $2.60 \mathrm{~m} / \mathrm{s}^{2}$ with a mean of $1.81 \mathrm{~m} / 2^{2}$.
\end{abstract}

Keywords-Wireless IMU, sprint start, accelerometer,sport, sensor.

\section{INTRODUCTION}

Throughout the lifecycle of the season [1], athletes and coaches work on different stages of a race at specific times. Whilst the number of stages in the sprinting event is not universally agreed upon by coaches ranging from three to six [2], in order to prevent over-complicating the process three principle phases have been defined:

1. Start phase - the point at which the athlete is in the set position to when both of their feet have left the starting blocks.

2. Drive / pick-up phase - from the end of the start phase until the athlete is standing in the fully upright running position.

\section{Maintenance phase - the remainder of the race.}

The first two phases, lasting around $40 \mathrm{~m}$ of a $100 \mathrm{~m}$ sprint, account for $64 \%$ of the final result [3]. Therefore, it is vital that coaches work to optimise these phases and monitor the progress closely.
Presently the technologies that allow an athlete's performance to be monitored are limited. The most accessible performance indicator that coaches use are the times their athletes record during competition or manual stopwatch recorded timings during training. The times recorded are hard to quantify as they do not provide any information as to what occurred within the race or training session. A small minority of coaches, and certainly only those at elite level, have the scientific resources (e.g. light gates [4]) enabling them to monitor performance features during training. Even with access to this type of technology the "complete" performance picture of the whole event is not provided and a large amount of post processing is required in terms of time, skills and costs. Expensive instrumented starting blocks (ISBs) have been successfully developed [5] and currently a form of ISB is used in the monitoring of false starts at world class events such as the IAAF Diamond League and the Olympic Games. However, these systems are constrained to feedback information on reaction time only. A cheap and easy to use device is therefore required to allow more people to benefit from accurate, relevant and detailed performance monitoring. During a sprint start an athlete aims to clear his / her starting blocks with as much horizontal force as possible in as short a period of time. Their rear foot is only in contact with the starting block for $45 \%$ of the time taken to complete phase 1 , thus their forefoot produces the majority of the force [6]. Wireless IMUs (inertial measurement units) have been found to give good temporal accuracy during walking and running [7]. An IMU has the capability to collect a large amount of information regarding transitional and rotational acceleration. The purpose of this study was to investigate the capability of an IMU in providing relevant and sufficiently accurate information for a sprint start performance and thus being used as cheaper an alternative solution to the more expensive ISBs.

\section{METHODOLOGY}

\section{A. Participants}

Five male amateur university athletes were recruited for the study (weight $=81 \pm 10.6 \mathrm{~kg}$, age $=20.6 \pm 0.5$ years old). All athletes were recruited from Loughborough University and gave their consent to take part. Throughout the trials the athletes wore their own footwear as spikes could not be worn within the laboratory setting. 


\section{B. Equipment}

Fourteen infrared Vicon T-Series cameras recorded 35 retro-reflective markers positioned on the athletes at $250 \mathrm{~Hz}$ using the Vicon Nexus system. The position of the markers is reported in Table I. Prior to testing the cameras were calibrated, so that the origin was known and thus positional data could be determined. The IMU used was a wireless device, developed at Loughborough University, consisting of a 3 -axis accelerometer and a 3-axis gyroscope. It was positioned on the athlete's lower back so that the $\mathrm{Z}$ axis of the IMU was parallel to the frontal plane of the athlete. Data were collected at $50 \mathrm{~Hz}$. Four retro-reflective markers were placed on the IMU to allow for its position to be tracked. Two wooden starting blocks, fixed at an angle of $45^{\circ}$ from the horizontal, were bolted to two independant Kistler force platforms. The force platforms collected data at $1000 \mathrm{~Hz}$ and were re-set to read a zero force before the start of each trial. Fig.1 shows the experimental set up.

\section{Protocol}

The athletes where instructed to carry out a warm up as they would for a regular training session. A period of familiarisation with the starting blocks was permitted. Once the athlete was ready the reflective markers were placed on their skin and the IMU was placed on their lower back. A static trial of each athlete in the "on your marks" position (Fig. 2.1) was recorded to allow for ease of Vicon post-processing. The athlete was then instructed to carry out a sprint start as they would in a race. The commands "on your marks", "set" (Fig. 2.2); and "go" were given. All recordings started on the "on your marks" command. The force plate and Vicon system were synchronised so that they stared recording at the same point. Each participant performed five sprint starts in total. Between each trail a period of recovery was allowed.

\section{Data Processing}

The Vicon data were reconstructed to determine position in three-dimensions. The static trial for each athlete was then manually labelled and this model was used to auto-label the remaining trials. Each trial was manually inspected for gaps and / or labelling errors. Any gaps of less than 50 frames were filled using the spline fill method, any gaps greater than 50 frames were filled with a pattern fill algorithm. A low pass $5^{\text {th }}$ order Butterworth filter with a cut off frequency of $14 \mathrm{~Hz}$ was selected as the optimal cut off frequency for the Vicon data. This was selected using the residual analysis technique [8].

TABLE I. POSITION OF MARKERS

\begin{tabular}{|l|l|l|l|}
\hline \multicolumn{3}{|l|}{ Markers on both left and right side } & Single marker \\
\hline Heel & $1^{\text {st }}$ Metatarsal & Top Shoulder & T10 \\
\hline Inside ankle & Outside ankle & Front shoulder & T2 \\
\hline Inside knee & Outside knee & Back shoulder & C7 \\
\hline Inside elbow & Outside elbow & Outside wrist & Clavicle \\
\hline Inside wrist & Pelvis & Back waist & Sternum \\
\hline
\end{tabular}

The IMU data were transformed into the global frame using the Euler angles transformation approach [9]. A low pass $5^{\text {th }}$ order Butterworth filter with a cut off frequency of $12 \mathrm{~Hz}$ was selected, again using the residual analysis technique [8].

The positional Vicon data was twice differentiated to get acceleration data. Only the data in the x-direction (athlete's horizontal movement) was investigated, in the first instance, for both the acceleration data from the IMU and the acceleration of the IMU itself, determined from the Vicon data. In order to synchronise the data, a moving Pearson's correlation algorithm was developed in Matlab. This algorithm used down sampled data (i.e. $50 \mathrm{~Hz}$ ) from Vicon. The Vicon data were correlated with the IMU acceleration data using a standard correlation algorithm. The maximum correlation coefficient was determined and the time stamp at this point was used to synchronise the two signals.

A script was written in Matlab 7.5.0 (R2007b) which automatically extracted eight key features from both Vicon and accelerometer traces following synchronisation. The frame number and the acceleration at each of these key features were determined and the mean and standard deviation calculated.

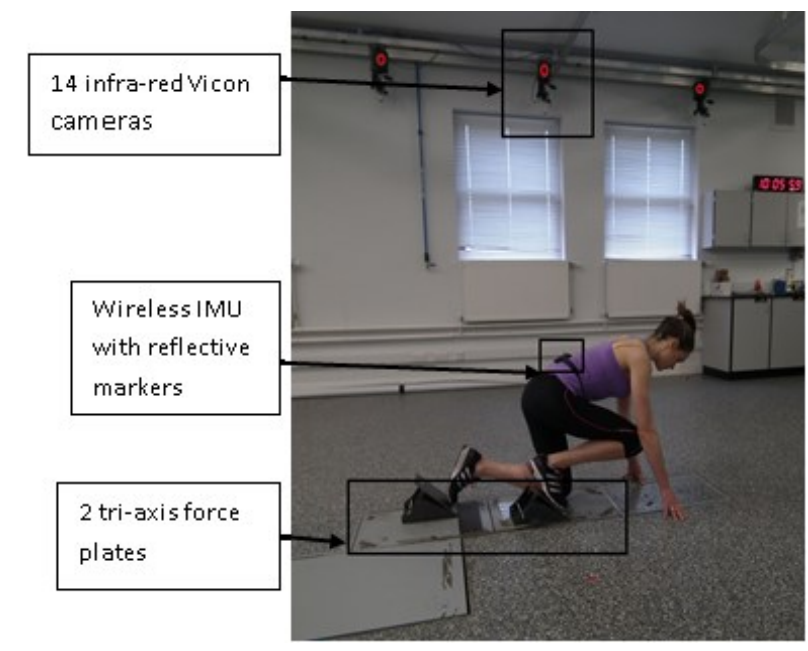

Fig.1 Experimental set up

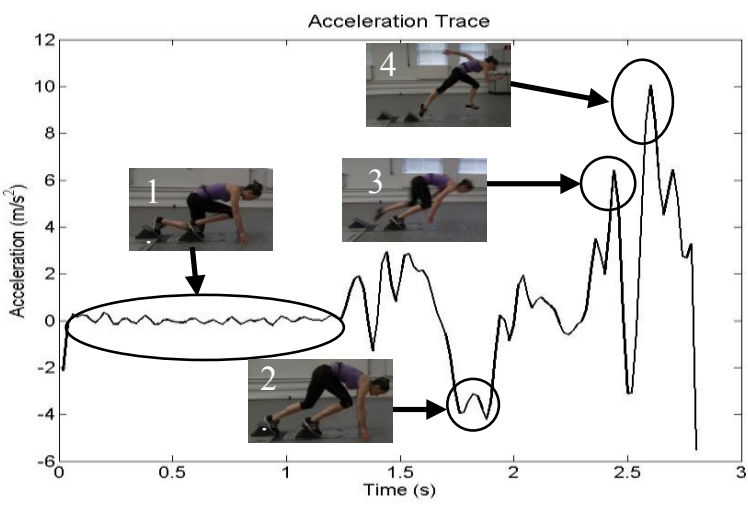

Fig 2. Sprint characteristics within the Vicon trace. 1. "on your marks", 2. "set', 3. "go" and 4. end of phase 1. 


\section{RESULTS AND DISCUSSION}

\section{A. Correlation}

An example of the key points of the sprint start that can be picked out of the Vicon trace are illustrated in Fig. 2 along with the images of the athlete's progression at these points. The results of the acceleration data collected by both the IMU and Vicon can be observed in Fig. 3. A Pearson's test for the two traces recorded from 25 trials results in a mean and standard deviation for the correlation coefficient of $0.907 \pm$ 0.068. This emphasises a positive correlation across all trials, suggesting that the IMU accelerometer data have represent the same features as the data recorded using the Vicon system.

\section{B. Timing}

Eight key features were automatically selected from each trace using the Matlab script. These features have been highlighted in Fig. 3 and the absolute mean and standard deviations between the IMU and Vicon for time and acceleration at these points listed in Table II. The results show that feature six is the most variable in terms of timing (i.e. $2 \pm 3.42$ frames) and feature eight (i.e. when the forefoot leaves the starting blocks) shows the least variability in time with the lowest standard deviation (i.e. $0.44 \pm 0.58$ ). Fig. 4 shows a graph of these data. The mean accuracy is 1.26 frames or $0.025 \pm 0.0214 \mathrm{~s}$.

\section{Acceleration}

The differences in acceleration recorded by the Vicon and IMU recorded results in a mean accuracy of $1.81 \mathrm{~m} / \mathrm{s}^{2}$ across each of the trials. The largest mean difference (i.e. $2.6 \mathrm{~m} / \mathrm{s}^{2}$ ) is seen for feature seven (i.e. following the rear foot leaving the starting blocks). Fig. 5 shows the mean difference in acceleration for each of the different features

\section{Systematic Error}

From the information illustrated in Fig. 4 and 5 the systematic error in the data collected using the IMU was investigated further. The mean of the timing and acceleration inaccuracies (1.28 frames and $2.33 \mathrm{~m} / \mathrm{s}^{2}$ respectively) highlight that a systematic error exists. The mean timing and acceleration accuracy for features seven and eight are the lowest, indicating that the features are characterised more robustly during increased changes in acceleration. To understand the systematic error in more detail a first order polynomial curve is fitted to these data (Fig 6), the line created has a gradient of 0.987 and intersects the $y$ axis at 0.0113 . Showing a very small systematic error in the acceleration data recorded using the IMU when compared to that recorded using Vicon.

The relative differences between the time and acceleration features are reported in Table III, the overall mean of $0.047 \pm$ 0.035 frames for time and $0.065 \pm 0.122 \mathrm{~m} / \mathrm{s}^{2}$ for acceleration illustrate that the accelerometer data is accurate in supplying relative information regarding each feature.

\section{CONCLUSION}

The IMU device has shown that the data collected has the ability to produce an accurate replication of a sprint start, as well as having a high degree of timing accuracy, within $0.06 \mathrm{~s}$ when compared to the gold standard. This makes it a much cheaper alternative to the currently available ISBs. If the relative differences between the identified features are used then any inherent systematic error in the IMU can be effectively removed. However the sprint start is a highly explosive movement and thus the current sampling rate of the IMU device (i.e. $50 \mathrm{~Hz}$ ) will mean high frequency variations will be missed. In order to be able to detect high frequency features a $2^{\text {nd }}$ generation IMU has been designed to be able to transmit IMU data at a frequency of $10 \mathrm{kHz}$. Positional accuracy determined from accelerometers is known to be limited by integration errors. However previous research [10] and [11] has shown it possible to correct for integration drift by using knowledge about when the IMU is stationary. The positive results suggest that with an increase in sampling rate and removal of the systematic errors within the data the IMU could be used to assist athletes and coaches in their pursuit to optimise performance.

An extra feature of this specific IMU allows data to be supplied to the user in real time, allowing the coach and athlete to receive meaningful, useful information that can be

TABLE II. MEAN AND STD DIFFERENCES OF TIMING AND ACCELERATION FOR EACH FEATURE.

\begin{tabular}{l|l|l|l|l|l|l|l|l|l}
\hline Feature & $\mathbf{1}$ & $\mathbf{2}$ & $\mathbf{3}$ & $\mathbf{4}$ & $\mathbf{5}$ & $\mathbf{6}$ & $\mathbf{7}$ & $\mathbf{8}$ & Overall \\
\hline Mean Frames difference & 1.48 & 1.4 & 1.28 & 1.48 & 1.36 & 2 & 0.8 & 0.44 & 1.26 \\
STD frames difference & 1.92 & 2.13 & 2.48 & 4.15 & 3.01 & 3.42 & 0.96 & 0.58 & 1.07 \\
Mean acceleration difference & 1.52 & 1.15 & 1.78 & 1.28 & 1.94 & 2.13 & 2.60 & 2.12 & 1.81 \\
STD acceleration difference & 2.04 & 1.18 & 1.60 & 1.35 & 1.62 & 2.02 & 3.09 & 1.74 & 1.47 \\
\hline
\end{tabular}

TABLE III MEAN DIFFERENCE BETWEEN TRIALS.

\begin{tabular}{l|l|l|l|l|l|l|l|l|l|l|l}
\hline Feature & $\mathbf{1}$ & $\mathbf{2}$ & $\mathbf{3}$ & $\mathbf{4}$ & $\mathbf{5}$ & $\mathbf{6}$ & $\mathbf{7}$ & $\mathbf{8}$ & Overall & STD \\
\hline Mean difference in time & 0.083 & 0.042 & 0.042 & 0.083 & 0 & 0.083 & 0.042 & 0 & 0.047 & 0.065 \\
Mean difference in acceleration & 0.03 & -0.038 & 0.022 & 0.071 & 0.079 & 0.109 & -0.079 & 0.33 & 0.035 & 0.122 \\
\hline
\end{tabular}




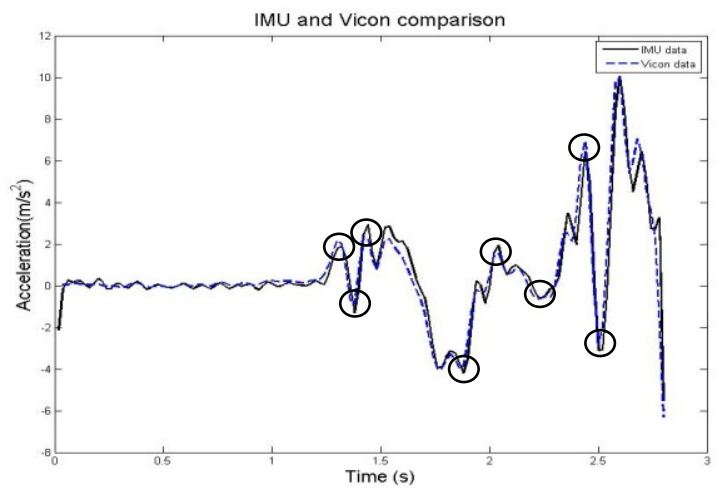

Fig. 3. Features automatically selected from each trial.

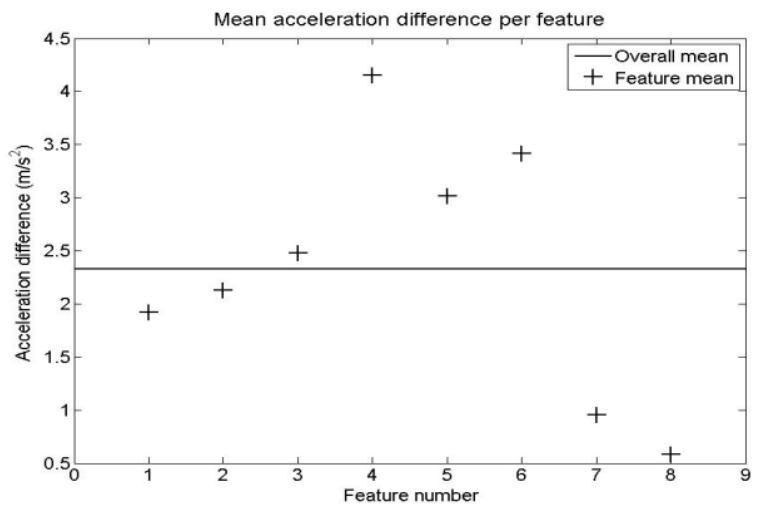

Fig. 5. Mean acceleration difference between IMU and Vicon features.

implemented into their performance with more effect than if it was given during a review session [12], which is a limiting factor in the majority of the currently used performance monitoring techniques.

Further work will include introducing a trigger into the system in order to allow coaches to determine reaction time, determination of the relevant features that enable the characterisation of the later phases of the sprint event and increasing the sampling frequency to reduce the current timing accuracy of approximately $0.025 \mathrm{~s}$

\section{REFERENCES}

[1] T. O. Bompa and M. Carrera, Periodization training for sport, $2^{\text {nd }}$ ed., Champaign, IL. Human Kinetics, 2005.

[2] R. Jones, I. Bezodis, and A. Thompson, "Coaching sprinting expert coaches' perception of race phases and technical constructs," Int. J. Sports Sci. Coach. Multiscience, 2009, vol 4, no. 3, pp. 385-396.

[3] T. Tellez, and D. Doolittle, "Sprinting from start to finish," Track Tech. Mountain View, 1984, vol. 88.

[4] L. Cheng, H. Tan, G. Kuntze, I. N. Bezodis, S. Hails, D. G. Kerwin and A. Wilson, 'A low cost accurate speed-tracking system for supporting

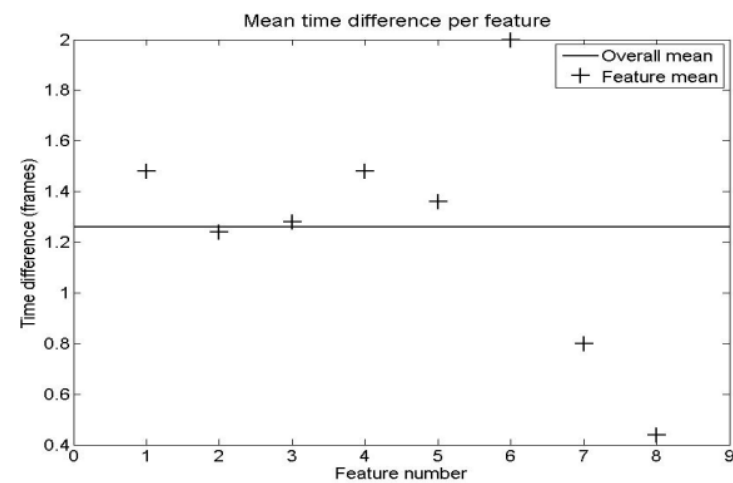

Fig. 4. Mean timing difference between IMU and Vicon features.

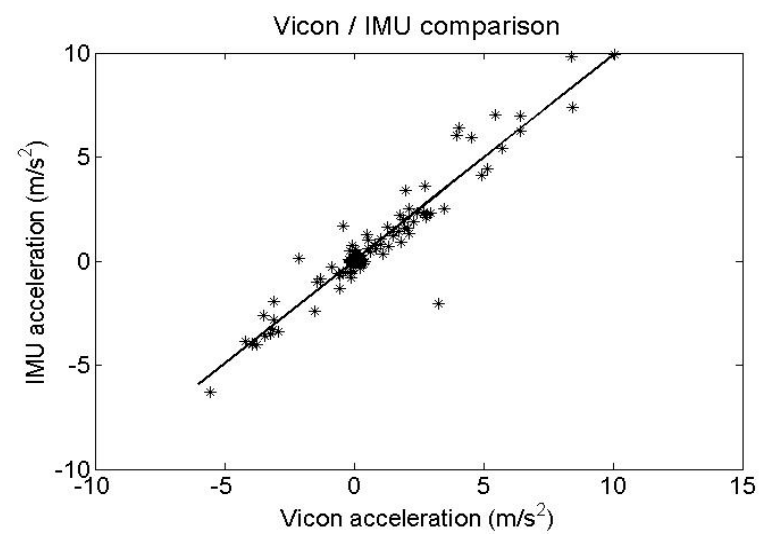

Fig. 6. Comparison of Vison vs IMU acceleration.

sprint coaching,' Proceedings of the institute of mechanical engineers, J. Sports. Eng. Technol, Professional engineering publishing, vol. 224, no. 2, pp.167-179, 2010.

[5] R. E. Gander, J. D. McClements, L. K. Sanderson, B. A. Rostad, K. E. Josephson, and A. J. Pratt, "Sprint start instramentation," IEEE Trans. Instrum. Meas. IEEE, 1994, vol. 43, no. 4, pp. 637-643.

[6] A. Mero, P. V. Komi, "Reaction time and electromyographic activity during a sprint start," Eur. J. Appl. Physiol. Springer, 1990, vol. 61, pp. 73-80.

[7] J. B. Lee, R. B. Mellifont, and B. J. Burkett, "The use of a single inertial sensor to identify stride, step and stance durations of running gait," J. Sci. Med. Sport. Elsevier, 2010, vol. 13 no. 2, pp. 270-273.

[8] D. A. Winter, Biomechanics of motor control of human movement, $4^{\text {th }}$ ed., Hoboken, NJ: Wiley, 2010.

[9] D. Titterton and J. Weston, Strapdown inertial navigation technology, $2^{\text {nd }}$ ed. Stevenage, IET. 2004.

[10] B. Mariani, C. Hoskovek, S. Rochat, C. Büla, J. Pender, and K. Aminiam, "3D gait analydid in young and elderly subjects using footworn inertial sensors," J. Biomech. Elsevier, 2010, vol. 43, no. 15, pp. 2999-3006.

[11] T. Le Sage, P. Conway, J. Cossor, S. Slawson, and A. West, "A wireless sensor system for monitoring the performance of a swimmers tumble turn," Proceedings of the institute of mechanical engineering. J. Sports Eng. Technol, USA, vol. 277, no. 3, pp. 161-171, September 2013.

[12] R. Kirby, "Development of a real-time performance measurement and feedback system for alpine skiers," Sports Technol. Taylor \& Francis, 2009, vol. 2, no. 2-1, pp. 43-52. 